\title{
Dual-Mode Elecromechanical Resonance of Nanobelts Observed by In-situ TEM
}

\author{
Z.L. Wang ${ }^{*},+$, X.D. Bai ${ }^{*}{ }^{* *}$, P.X. Gao ${ }^{*}$ and E.G. Wang ${ }^{* *}$
}

* School of Materials Science and Engineering, Georgia Institute of Technology, Atlanta, Georgia 30332-0245

** International Center for Quantum Structures and State Key Laboratory for Surface Physics, Institute of Physics, Chinese Academy of Sciences, Beijing 100080, China

+e-mail: zhong.wang@mse.gatech.edu

Semiconducting oxide nanobelts [1] and nanowires [2] have shown novel electronic, chemical and optical properties $[3,4]$. The nanobelts have a distinct structural morphology, characterized by a rectangular cross-section and a uniform structure, which could be directly used as nanocantilevers and nanoresonators in nanoelectromechanical systems (NEMS) [5,6] (Fig. 1A). A key phenomenon for applying nanobelts in NEMS technology is their electromechanical resonance behavior, and an important physical quantity for cantilever applications is their bending modulus. In this paper, the electromechanical resonance of a single $\mathrm{ZnO}$ nanobelt, induced by an alternative electric field, was studied by in-situ transmission electron microscopy (TEM). Due to the rectangular cross-section of the nanobelt, two fundamental resonance modes have been observed in corresponding to the two orthogonal transverse vibration directions. The electromechanical resonant behavior of the nanobelts is directly correlated with their distinct structural feature, showing a possibility for versatile applications as nanoresonators and nanocantilevers.

A new experimental approach, based on the electric-field-induced resonant excitation, has been developed for direct measurement the mechanical properties of individual nanowire-like structures by in-situ TEM [7,8]. Using this method, mechanical properties of carbon nanotubes [7], silicon nanowires [9], and silicon carbide-silica composite nanowires [10] have been investigated. This is the technique that will be applied to study the resonance behavior of individual $\mathrm{ZnO}$ nanobelts. From the classical elasticity theory for a rectangular beam [11], the fundamental resonance frequencies corresponding to the thickness direction (x-axis) and width direction (y-axis) (Fig. 1B), respectively, are

$$
v_{x i}=\frac{\beta_{i}^{2} T}{4 \pi L^{2}} \sqrt{\frac{E_{x}}{3 \rho}} \quad \text { and } \quad v_{y i}=\frac{\beta_{i}^{2} W}{4 \pi L^{2}} \sqrt{\frac{E_{y}}{3 \rho}},
$$

where $\beta_{i}$ is a constant for the $i$ th harmonic: $\beta_{1}=1.875$ and $\beta_{2}=4.694, E_{x}$ is the bending modulus for the vibration along the x-axis, $L$ is length of the nanobelt, $\rho$ is mass density; and $E$ the corresponding resonance frequency. The ratio of the two fundamental frequencies is directly related to the aspect ratio of the nanobelt by $v_{y 1} / v_{x 1}=W / T\left(E_{y} / E_{x}\right)^{1 / 2}$.

Due to the rectangular cross-section of the nanobelt, two fundamental resonance modes have been observed in corresponding to two orthogonal transverse vibration directions (Fig. 2), showing the versatile applications of nanobelts as nanocantilevers and nanoresonators. The bending modulus of the $\mathrm{ZnO}$ nanobelts was measured to be $\sim 52 \mathrm{GPa}$ (Table I). The two resonance modes just correspond to the two modes of the tip operation when the nanobelt-base cantilever is used as a force sensor: one 
is the tapping mode, and the other is the non-contact mode. Thus, the force sensor fabricated using the $\mathrm{ZnO}$ nanobelts is versatile for applications on hard and soft surfaces [12].

[1] Z.W. Pan, Z.R. Dai, and Z.L. Wang, Science 291, 1947 (2001).

[2] M.H. Huang, S. Mao, H. Feick, H. Yan, Y. Wu, H. Kind, E. Weber, R. Russo, and P. Yang, Science 292, 1897 (2001).

[3] M.S. Arnold, P. Avouris, Z.W. Pan, and Z.L. Wang, J. Phys. Chem. B 107, 659 (2002).

[4] E. Comini, G. Faglia, G. Sberveglieri, Zhengwei Pan, and Zhong L. Wang, Appl. Phys. Lett. 81, 1869 (2002).

[5] H.G. Craighead, Science 290, 1532 (2000).

[6] W. Hughes and Z.L. Wang, Appl. Phys. Letts. submitted (2002).

[7] P. Poncharal, Z.L. Wang, D. Ugarte, and W.A. de Heer, Science 283, 1513 (1999).

[8] Z.L. Wang, P. Poncharal, and W.A. de Heer, Pure Appl. Chem.72, 209 (2000).

[9] Z.L. Wang, Adv. Mater. 12, 1295 (2000).

[10] Z.L. Wang, Z.R. Dai, Z.G. Bai, R.P. Gao, and J. Gole, Appl. Phys. Lett. 77, 3349 (2000).

[11] L. Meirovich, Elements of Vibration Analysis, (McGraw-Hill, New York, 1986).

[12] We acknowledge support from the NASA URETI program (E-16-V14), China NSF, International Center for Quantum Structures (CAS) and Georgia Tech.

Table I. Bending modulus of the $\mathrm{ZnO}$ nanobelts. $E_{x}$ and $E_{y}$ represents the bending modulus corresponding the resonance along the thickness and width directions, respectively.

\begin{tabular}{cccccccc}
\hline & $\begin{array}{c}\text { Length } \\
L(\mu \mathrm{m})\end{array}$ & $\begin{array}{c}\text { Width } \\
W(\mathrm{~nm})\end{array}$ & $\begin{array}{c}\text { Thickness } \\
T(\mathrm{~nm})\end{array}$ & $\begin{array}{c}\text { Resonance Fequency } \\
(\mathrm{kHz})\end{array}$ & \multicolumn{2}{c}{$\begin{array}{c}\text { Bending Modulus } \\
(\mathrm{GPa})\end{array}$} \\
\hline Nanobelt & $( \pm 0.05)$ & $( \pm 1)$ & $( \pm 1)$ & $v_{x 1}$ & $v_{y 1}$ & $E_{x}$ & $E_{y}$ \\
1 & 8.25 & 55 & 33 & 232 & 373 & $46.6 \pm 0.6$ & $50.1 \pm 0.6$ \\
2 & 4.73 & 28 & 19 & 396 & 576 & $44.3 \pm 1.3$ & $45.5 \pm 2.9$ \\
3 & 4.07 & 31 & 20 & 662 & 958 & $56.3 \pm 0.9$ & $64.6 \pm 2.3$ \\
4 & 8.90 & 44 & 39 & 210 & 231 & $37.9 \pm 0.6$ & $39.9 \pm 1.2$ \\
\hline
\end{tabular}
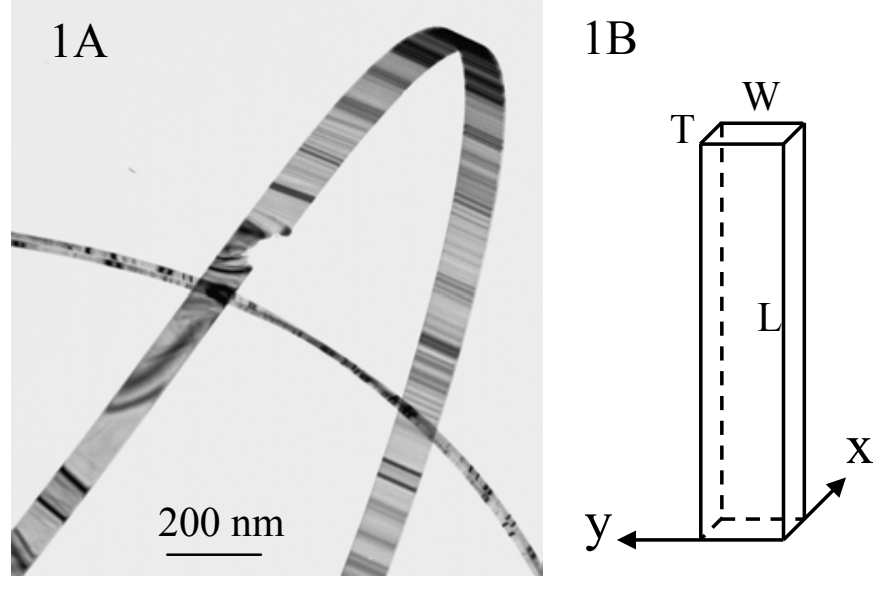

$2 \mathrm{~A}$

2B

FIG. 1. (A) A typical TEM image of a $\mathrm{ZnO}$ nanobelt. (B) Geometrical shape of a nanobelt. FIG. 2. A selected $\mathrm{ZnO}$ nanobelt at (A) the first harmonic resonance in $\mathrm{x}$ direction, $v_{x 1}=622 \mathrm{kHz}$, and (B) the first harmonic resonance in y direction, $v_{y 1}=691 \mathrm{kHz}$. 
https://doi.org/10.1017/S143192760344169X Published online by Cambridge University Press 